\title{
A VALIDATION STUDY FOR A NEW EROSION MODEL TO PREDICT EROSIVE AIRFOIL DEFOULING
}

\author{
ARTHUR RUDEK ${ }^{1,2}$, DAVID MUCKENHAUPT ${ }^{1}$, THOMAS ZITZMANN $^{1}$, \\ GERALD RUSS ${ }^{1} \&$ BARRY DUIGNAN $^{2}$ \\ ${ }^{1}$ Hochschule Darmstadt, University of Applied Sciences, Germany. \\ ${ }^{2}$ Technical University Dublin, Ireland.
}

\begin{abstract}
A new defouling erosion model for Lagrangian particle tracking is used to predict defouling of amorphous, heterogeneous coatings such as those typically found in aircraft compressors. The main problem description, the mathematical formulation and the underpinning experiment of the model are presented in a previous communication by the authors. In this work, the Ansys CFX implementation of the model is described and an experiment is presented for the validation of the model. Air flows laden with a number of dry-ice particles are observed in an optically accessible stream channel containing a flat plate target. The defouling process of these particles is recorded with HSCs and the main parameters, such as indentation size in fouling layers, are processed and compared to corresponding numerical results. The model parameters considered are particle impact velocity and angle as well as particle and fouling material. Typical coatings which are relevant to commercial aircraft defouling processes are investigated. The target plate angle and the air velocity are varied and dry-ice particles of random size and shape are injected into the flow. The experiment is set up in a wind-tunnel test-rig and all recordings are made using two HSCs, a digital camera and Prandtl probe measurement. Experimental and numerical defouling results show good overall agreement for steep target angles but significant deviations for low target angles. Potential improvement to the defouling erosion model is discussed based on these results. The model as presented is used in large-scale compressor defouling simulations in the development process of on-wing aircraft maintenance systems.
\end{abstract}

Keywords: aircraft engine defouling, $\mathrm{CO}_{2}$ dry-ice blasting, solid particle erosion, validation experiment.

\section{INTRODUCTION}

On-wing aircraft engine cleaning is a current topic of research for commercial aircraft operators. Engine maintenance cost represents approximately $35 \%$ to $40 \%$ of an airline's total maintenance cost [1]. Periodic on-wing engine cleaning results in greater operating efficiency and lower emission rates. In this work compressor defouling is addressed. Compressor fouling is mainly caused by in-service ingestion and deposition of various types of solid and fluid foulants from ambient air, such as unburned hydrocarbons, insects, soil, salt, etc. [2-4]. This leads to decreased engine efficiency and power output and higher fuel consumption, pollutant emission and increased operational cost [3, 5-10]. To counteract this, a number of aircraft engine compressor defouling systems have been developed in recent decades. These are mostly based on solid (e.g. coal-dust, nut-shells) or liquid (e.g. water droplets, solvents) particle injection into the engine core while the engine is dry cranked [10-13]. The most recent research at Hochschule Darmstadt (hda) and Dublin Institute of Technology (DIT) in cooperation with Lufthansa Technik AG (LHT) resulted in the new Cyclean 2.0 cleaning system which is based on pressurized air which carries dispersed $\mathrm{CO}_{2}$ dry-ice particles. The particles clean the compressor blades by erosive wear. The basic principles of the system are described in [14]. Further details of the system including particle laden in-engine flow investigations and simulations are described by the authors in $[15,16]$ and the entire study dealing with numerical simulations of the novel Cyclean 2.0 cleaning procedure is described in detail in [17]. 


\section{STATE OF THE ART}

The numerical simulation of the new Cyclean 2.0 cleaning system operating with dry-ice was one of the key goals in the research mentioned above. The simulations must be carried out with the commercial numerical code ANSYS CFX and must incorporate an appropriate erosion prediction formulation. The Ansys CFX code incorporates a number of erosion models which are typically used in turbomachinery, such as those from FINNIE [18] and GRANT and TABAKOFF [18]. However, an extensive literature review, most of which was presented in RUDEK et al. [15] by the authors, revealed that no erosion model is available at present, which is capable of predicting the erosion of amorphous and heterogeneous coating materials, such as fouling layers typically found on aircraft engine blades.

To address this, the new energy-based erosion model introduced by the authors in Rudek et al. [15] was developed. It uses the experimental "Dynamic Indentation Testing" (DI) in order to determine the behavior of fouling material under erosion during engine cleaning. Several other researchers have taken a comparable approach to determine the erosion of technical coatings, such as paint, and their work is summarized in detail in Rudek et al. [15] and briefly described below.

The new defouling erosion model considered in this work is based on an energy balance comparable to the dynamic hardness definition by Sundararajan et al. [19]

$$
H d:=\frac{\frac{1}{2} \cdot m_{P} \cdot\left(v_{p, 1}^{2}-v_{p, 2}^{2}\right)}{V_{i m p}}=\frac{1}{2} \cdot \frac{m_{P}}{V_{i m p}} \cdot v_{P, 1}^{2} \cdot\left(1-\varepsilon^{2}\right)
$$

who introduced an energy-based model to predict crater volume $V_{I M P}$ and particle rebound characteristics (described by the particle mass $m_{P}$ and its velocity before $v_{P, 1}$ and after impact $v_{P, 2}$ ) in solid material erosion processes. They used the coefficient of restitution in eqn. (1)

$$
\varepsilon=\frac{v_{P, 2}}{v_{P, 1}}
$$

to assess the energy consumed by the particle-wall interaction.

The defouling erosion model presented is underpinned with data acquired by means of single particle experiments comparable to the DI testing procedure presented by Hutchings et al. [20, 21] and Sundararajan et al. [19, 22]. From this experiment, it is possible to determine the amount of energy necessary to penetrate and remove certain portions of typical foulants from aircraft compressor airfoils and to predict the amount removed. The experiment is designed under the constraints of the main conditions for DI testing reported in $[19,22]$, which are

- quasi-static impact behavior,

- negligible stress-wave energy losses,

- negligible particle rotation,

- particle hardness must be greater than target (i.e. fouling) hardness,

- superposition of erosion from normal and tangential forces is possible.

Following $[20,21,23,24]$ it is assumed that the defouling process is independent of particle material and therefore reference material particles, which do not disintegrate on impact, 
are used for rebound testing. The results are adapted to dry-ice particles using empirical defouling functions. A procedure comparable to this was reported and extensively examined by PAPINI and SPELT in their decoating studies [23, 25, 26].

Gondret et al. [27, 28] investigated various material pairings in a range of particle impact tests and reported similarity in their restitution behavior if the coefficient of restitution is described as a function of the near-wall Stokes number

$$
S t_{c}=m_{P} \cdot \frac{v_{P}}{6 \cdot \pi \cdot \eta_{c} \cdot r_{P}^{2}}=\frac{1}{9} \cdot \frac{\rho_{P} \cdot v_{P} \cdot d_{P}}{\eta_{c}}
$$

which was derived from the particles ODE of motion in near wall formulation only considering viscous forces (index: $\mathrm{c}$ for coating) and which incorporates the influence of particle size (radius $r$ and diameter $d$ ), mass $\rho$, and velocity $v$.

A similar procedure is used in the underpinning experiment of the erosion model used in this work to measure foulant properties with non-disintegrating particles made from reference material and to adapt these findings to dry-ice particles. Comparable normalization approaches have been reported in [29, 30].

Based on the findings reported in $[25,29-31]$ it was expected that the energetic properties of the defouling process are measurable only in a certain range of normal impact velocities, which was demonstrated in [17]. Furthermore, there various angular dependencies of the defouling rates are considered for various fouling materials following [32]. Brittle and ductile material behavior was taken into account when the impact angles were chosen for the new experiment (i.e. $90^{\circ}$ and $30^{\circ}$ measured parallel to the wall), following for example [33, 34].

\section{MODEL DESCRIPTION}

The following model description represents a modified formulation of what was reported in [15] by the authors and it is adapted from RUDEK [17]. The model calculates particle energy dissipation during fouling erosion and measures defouling.

Basically following Gondret et al. [27, 28], the process is assumed to be dependent on the near-wall Stokes number, eqn. (3) but it utilizes the viscosity of ambient air instead of the viscosity of the coating. The formulation

$$
\delta_{e}^{\{p a r t, f o u, a\}}=\frac{\rho_{\text {ref }}}{2} \cdot \delta \varepsilon^{\{f o u, 0\}} \cdot\left(v_{1}^{\{r e f E Q, 0\}}\right)^{2} \cdot F_{I M P}^{\{\text {part }, \text { fou }, a\}}
$$

is used to describe the energy dissipation $\delta \varepsilon^{\{f o u, 0\}}$ of a certain particle on impact upon a fouled target and to assess the proportion of defouling energy $\delta e^{\{p a r t, f o u, \alpha\}}$, which is necessary to indent and to remove a proportion of fouling. It is adapted from the dynamic hardness definition, eqn. (1).

Defouling energy $\delta e$ is related to empirical restitution data from reference particle material impacts (index: ref) and, if necessary, is scaled with a defouling relation $F_{I M P}$ to any particle material. The superscript is important to this formulation and it reads as follows:

- $p a r t=$ particle material (i.e. $\operatorname{refE} Q=$ reference material at equivalent velocity),

- $f o u=$ fouling material,

- $\alpha=$ impact angle (i.e. $0=$ normal to the wall). 
The product of the first three contributors to the right-hand side of eqn. (4) describes reference material dissipation at dry-ice equivalent velocity. It is used to consider the difference in dissipated energy from impacts of non-disintegrating reference material particles in the normal direction on clean and fouled targets

$$
\delta \varepsilon^{\{f o u, 0\}}:=\left[\left(\varepsilon^{\{0\}}\right)^{2}-\left(\varepsilon^{\{f o u, 0\}}\right)^{2}\right]
$$

and $\delta \varepsilon$ is defined to be the impact dissipation factor. It is derived from experimental data of reference particle rebounds measured in the normal direction to the wall's surface (superscript: 0). Therefore the normal component of reference material particle impact velocity, which is normalized to a dry-ice equivalent (superscript: ref $E Q$ ) is used in eqn. (4). This dry-ice equivalence is derived from Stokes-number comparison of the investigated particles (here dry-ice) to those made from reference material. The variable $\delta \varepsilon$ is assumed to be dependent on fouling material (superscript: fou) only.

The fourth contributor to the right-hand side of eqn. (4) represents the scale function

$$
F_{I M P}^{\{\text {part }, f o u, a\}}=\left(\frac{d_{I M P}^{*\{\text { part }, \text { fou }, a\}} \cdot d_{P}^{\{\text {part }\}}}{d_{I M P}^{*\{\text { part }, f o u, 0\}} \cdot d_{P}^{\{\text {ref }\}}}\right)^{2}
$$

and its superscript indicates that it is a function of particle material (part), fouling material (fou) and impact angle $(\alpha)$. The purpose of this function is to scale the proportion of defouling energy calculated by means of the dissipation factor. This dissipation factor is assumed to be dependent on the proportion of fouling removed from reference material indentations and the function above is used to account for actual proportions of defouling energy consumed to indent the same fouling material by any particle material at any impact angle.

Both areas (i.e. that defouled by reference material and that defouled by the actual material investigated) are calculated by means of the experimentally correlated indentation diameter

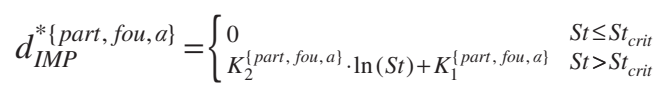

with the correlation coefficients $K_{1}$ and $K_{2}$. The onset of erosion for the particle-fouling combination under consideration is described by the critical Stokes number $S t_{c r i t}$.

The quotient described from eqn. (6) is used to scale the dissipated portion of energy consumed by the defouling from reference material ( $r e f$ ) values to actual material (part) values. The defouled area $A_{I M P}$ from one single particle impact is consequently calculated:

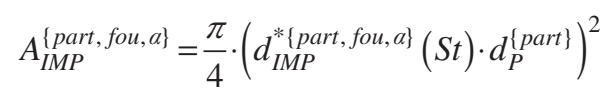

\section{EXPERIMENTAL SET-UP}

A wind-tunnel experiment is designed in order to create an optically accessible validation scenario towards the new defouling erosion model and this set-up is shown in Fig. 1 with the main dimensions. This testing section is directly flanged to the nozzle of the wind-tunnel, which delivers the air flow (a) at various air velocities. 

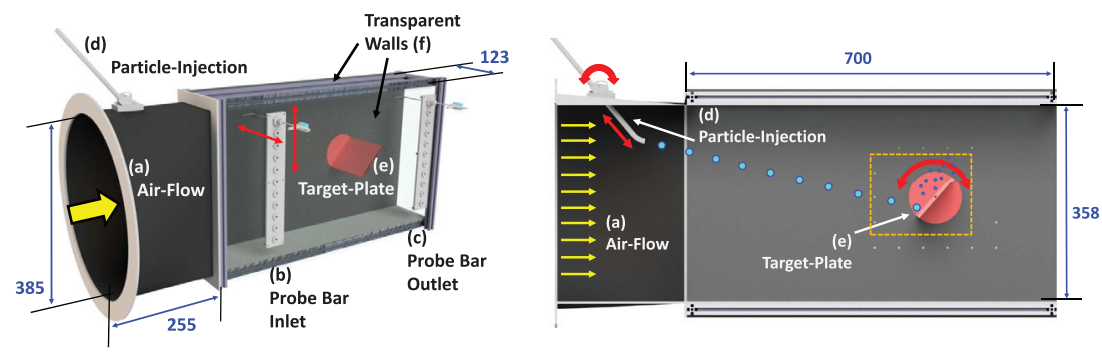

Figure 1: Experiment for numerical validation: schematic (left) and section view (right).

Probe bars (b) and (c) are used for the positioning of Prandtl probes with integrated thermocouples (type K) and these are located at the inlet and the outlet of the rectangular shaped main part of the testing section. These probes are used to measure flow properties such as pressure, velocity and temperature. Flow profiles can be recorded in both horizontal and vertical directions, as indicated by the red arrows in the figure. A number of particles can be introduced into the air-flow via the tubular injection system (d) and these are transported by the flow and impact upon the target plate (e).

The vertical and angular positions of the injection tube as well as the angle of the target plate can be varied, as indicated by the red arrows in Fig. 1, right. The testing section is optically accessible through transparent upper and side walls (f) which make the utilization of HSCs possible for tracking and sizing of primary and secondary dry-ice particles. An exchangeable target plate is used for defouling tests (not shown) with which defouling action is measured after a number of particle impacts. To achieve this, images of the target plate surfaces are recorded before and after particle impacts outside the testing section and these are compared by image post-processing.

\section{MAIN RESULTS}

Initially a pure air-flow run-up study is carried out and a representative selection of results is presented here. Furthermore, the most important findings of the final particle laden flow simulations are highlighted. The whole study presented in [17] comprises a systematic grid study, the discussion of a symmetry assumption and the extensive comparison of numerical to experimental results for air flow properties. Based on this, the numerical set-up for the main validation case investigations of the newly developed models is chosen. A parameter discussion is presented to determine the predictive capabilities of the models in conjunction with Ansys CFX simulations. Experimental data are recorded for defouling erosion and numerical results are compared to experimental data.

It is assumed that the behaviour of the validation experiment can be numerically predicted by considering a mid-plane cut through the rectangular part of the experimental set-up, assuming periodical symmetry at its sides. This assumption is based on preliminary experimental observations of POM and dry-ice particle tracks and flow field measurements. The particles are injected at the mid-channel and in all cases considered they impact the target in the central $33 \%$ of the channel. In order to show that side wall effects do not significantly influence the mid-plane flow, flow parameters were measured at a grid of locations across a number of vertical and horizontal positions across the section at the inlet and at the outlet planes of the channel and the results from this study are presented in detail in Rudek [17]. 


\subsection{Pure air flow validation}

In this section, results from steady-state RANS air flow simulations are compared to experimental data recorded in the mid plane of the channel at all vertical positions. Figure 2 shows simulation results of the two most extreme flow conditions (i.e. case 1 with lowest velocity and lowest target angle and case 4 with highest velocity and highest target angle).

Contours of static pressure are projected to the rear symmetry plane of the numerical volume and velocity streamlines are drawn from the inlet to the outlet plane. A wake region is clearly visible for both cases and the target influence upon the pressure field is also clearly visible. A high forebody and low afterbody pressure field is found to establish and it is mainly influenced by the air velocity and the target plate angle.
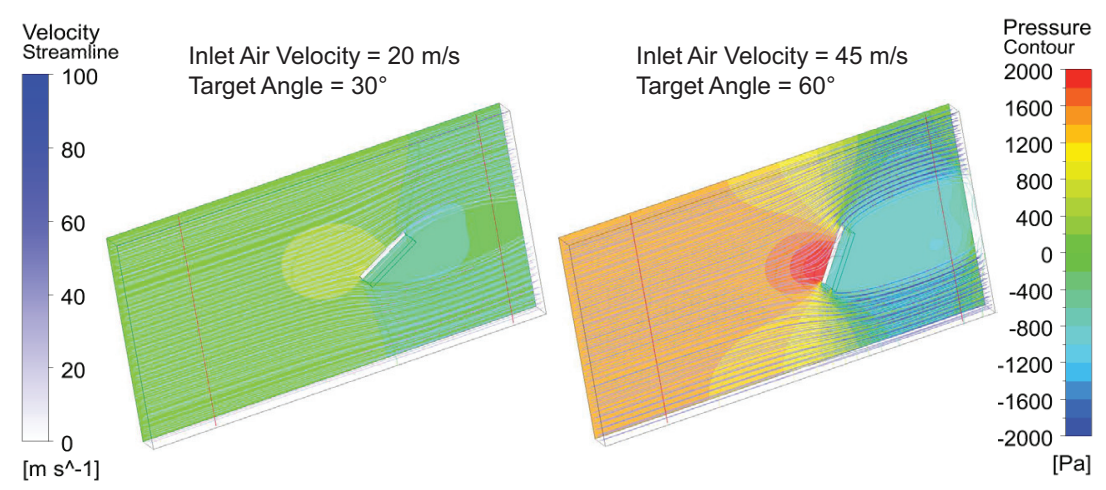

Figure 2: Numerical results for pure air flow in mid-plane symmetry volume for case 1 (left) and case 4 (right) - velocity streamlines and contours of static pressure are shown (Note: steady state RANS simulations cannot capture possible velocity fluctuations downstream the target plate, only the mean flow pattern is predicted).

A typical comparison of pressure and velocity profiles at the afterbody measurement plane (both forebody and afterbody measurement positions are indicated by the red vertical lines in Fig. 2 is shown in Fig. 3 for case 4. The predicted pressure and velocity trends are comparable to the experimental data and the mean deviations between predicted and experimental data
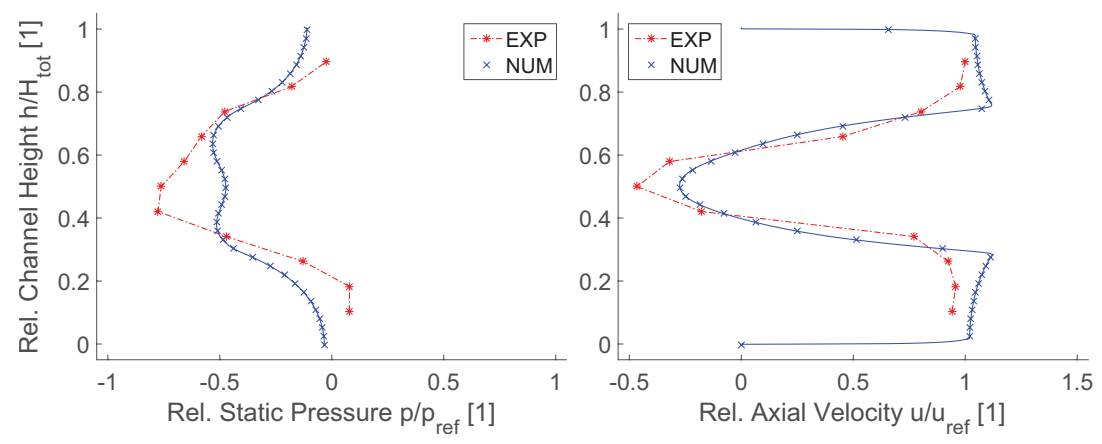

Figure 3: Case 4: air flow pressure (left) and velocity (right) profiles at the outlet plane - comparison of numerical to experimental data. 
are $12 \%$ for the pressure profile and $16 \%$ for the velocity profile. A more detailed discussion of this air-flow validation study is presented in Rudek [17]. Based on these results the set-up is assumed to be valid for the later particle model validation simulations.

\subsection{Particle tracking validation}

In the second step of the run-up study, experiments and simulations using polyoxymethylene (POM, i.e. used as reference material) particles are compared to assess the ability of the simulation to predict particle transportation and impact behavior. Particle injection is implemented in the numerical set-up by setting the initial particle velocity vector and its position in vertical direction at the inlet boundary corresponding to data measured in the experiment.

Whilst the continuous air flow is simulated by means of the Euler approach using the energy equation and Newtons material law, the dispersed particle phase is simulated by means of Lagrangian particle tracking. Therefore, the particle ODE of motion

$$
m_{P} \cdot \frac{d^{2} \mathrm{X}_{P}}{d t^{2}}=\sum_{i=1}^{n} F_{i}
$$

is solved in the area of interest. It relates particle inertia forces (represented by its mass $m_{P}$ and its acceleration) to the sum of $n$ external forces $\mathrm{F}_{i}$ acting on this particle.

Figure 4, left, shows a montage of typical experimental recordings of a POM particle with a diameter of $3.0 \mathrm{~mm}$ at various instants of time pre- and post-impact. The corresponding pre- and post impact angles of the particles are measured with respect to the horizontal plane of the set-up (indicated in the figure). Hence, negative angle values indicate negative vertical particle velocity components. The particle velocity is postprocessed with the recordings using in-house developed procedures such as those presented in $[17,35,36]$.

The comparison of the data from the run-up study with various POM particles is displayed in Fig. 4, right. The diagram shows the pre- and post-impact flight path angles of the particles (i.e. measured to the horizontal as explained in the discussion above) and the absolute
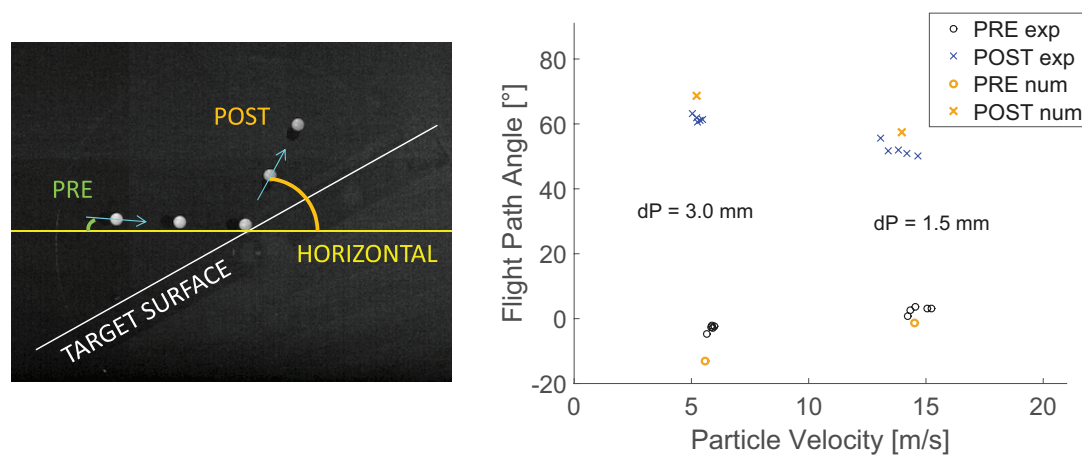

Figure 4: Montage of typical HSC POM particle track recordings at various pre- and post- impact instants of time (left) and comparison of numerical and experimental POM particle tracking results - particle impact behavior (right). 
pre- and post-impact velocity values from both numerical and experimental results. Satisfactory agreement between numerical and experimental data is achieved with the simulation set-up chosen.

Particle velocities prior to and after the instant of collision with the target plate are found to be precisely predicted. The impact angles are underpredicted and, as a consequence, the outbound angles are overpredicted. These deviations are more significant for larger particles at lower velocities. Possible causes for the deviations are the simplifications of the simulation assuming constant coefficients of restitution, such as given in eqn. (2), and neglecting rotation of the particles.

The overall agreement of all numerical data compared to experimental results is satisfactory. The mean deviations of the particle velocities range from $1 \%$ to $5 \%$ and these of the angles range from $5 \%$ to $12 \%$. Parts of the study were carried out with the opening boundary condition at the outlet of the numerical control volume and this leads to more significant deviations in the pressure profiles predicted at this position which is discussed in detail in [17]. However, the particle tracks seem to be independent of these deviations and it can therefore be concluded that the set-up chosen is adequate for all validation cases considered.

\subsection{Defouling erosion validation}

The set-up presented above is assumed to be valid for the prediction of the main validation situations presented here and it is used to assess the predictive capabilities of the CFX implementation of the new defouling erosion model. A grid is used which gives results independent from spatial discretization and the mid plane cut is applied because the above study showed no influence of the side walls upon the particle tracks in the middle of the experimental set-up. In addition, the boundary conditions applied showed no negative influence upon the predicted particle tracks.

If the dry-ice particles collide with solid walls they disintegrate into smaller fragments and a proportion of the fouling is removed from the airfoil. To account for this breakup process in the simulations, an experimentally based particle breakup model and the new defouling erosion model for dry-ice particles have been developed and the basic assumption of both models is an energy balance. Mass

$$
m_{P}=\sum_{j=1}^{k} m_{i}+\delta m_{s u b}
$$

and kinetic energy (index kin)

$$
E_{P, k i n}=\sum_{j=1}^{k}\left(E_{i, k i n}+E_{i, b u}\right)+E_{s u b}+E_{e r}
$$

of the impacting particle (index $P$ ) are conserved by balancing the impacting particle variables with those of the $k$ secondary particles in the new numerical breakup procedure.

All dispersed secondary particles and the sublimated proportion of primary particle mass (index $s u b$ ) are considered in the mass balance, eqn. (10). The energy balance, eqn. (11), accounts for the kinetic energy, the breakup energy (index $b u$ ), the sublimated energy proportion and the energy proportion used for the defouling erosion (index er) on its right-hand side. The last contribution is derived directly from the dissipated energy portion, eqn. (4), of the 
new erosion model. More detailed descriptions of the new particle breakup model can be found in $[16,17]$ and will be reported by the authors in an additional future communication.

In the validation experiment a HSC is used to record the primary particles impacting the target plate. An exchangeable target surface is used, which is coated with either PTFE or SALT and photographed before starting the experiment. After a certain number of primary particle impingements (i.e. 30-50 per parameter) the target plate is removed and its partially defouled surface is photographed again. A before-after comparison, comparable to what is described in RUDEK et al. [15], delivers the desired defouling statistics.

Numerical simulations are carried out of this validation situation and the primary particle injection position, velocity, direction and size distribution are derived from experimental measurements. The experimental parameters used for the defouling tests are approximately 25 and $50 \mathrm{~m} / \mathrm{s}$ nominal air velocity as well as $30^{\circ}$ and $60^{\circ}$ nominal target angle. The two artificial fouling materials, PTFE and SALT, are used for each parameter.

This results in a total of 8 tests, each of which was experimentally carried out twice. In the corresponding numerical simulations a total of 30 primary particles was considered for each parameter. Typical results from numerical defouling predictions are presented in Fig. 5: the left-hand image shows little defouling of the SALT layer in the case of $25 \mathrm{~m} / \mathrm{s}$ nominal air velocity and $30^{\circ}$ target angle compared to significant defouling of PTFE with $45 \mathrm{~m} / \mathrm{s}$ nominal air velocity and $60^{\circ}$ target angle.
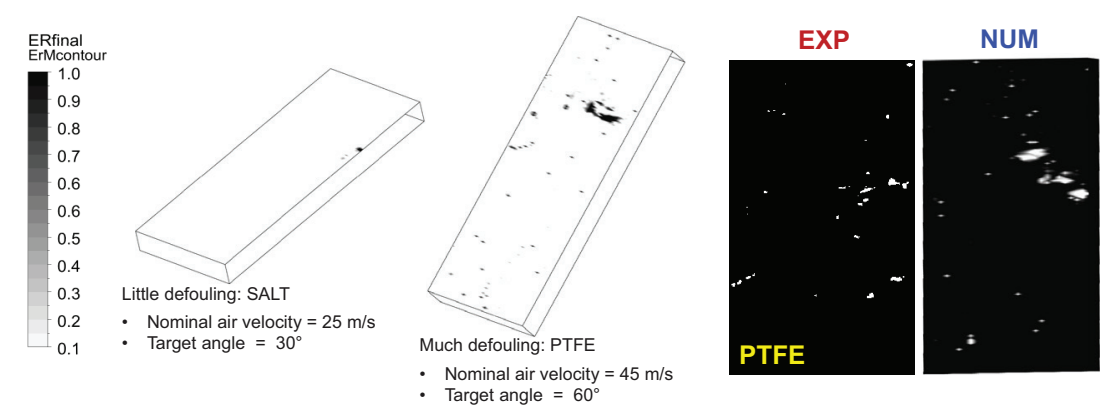

Figure 5: Typical indentation pattern from defouling simulations - little defouling of SALT coating and significant defouling of PTFE coating (both left); qualitative comparison of typical indentation pattern from predicted and experimental defouling of PTFE with the color map inverted (right).

A qualitative comparison of predicted defouling to experimental results is shown in Fig. 5, right, and a typical pattern from PTFE coating is displayed. The comparability of the defouling pattern can be seen. From this comparison it becomes clear that secondary particle indentations play a key role in PTFE defouling. This is not the case for SALT (here without display). For this reason the first comparison of numerical to experimental data deals with the number of indentations per primary particle and this is presented in Fig. 6.

The left-hand display shows results from PTFE testing and the right-hand display those from SALT layer investigations. Both diagrams show mean values from 8 experiments and 4 corresponding simulations. Increasing the primary particle Stokes numbers increases the number of indentations per particle and this can be seen for both PTFE and SALT layers. The mean values for PTFE are higher compared to those for SALT, which indicates that secondary 

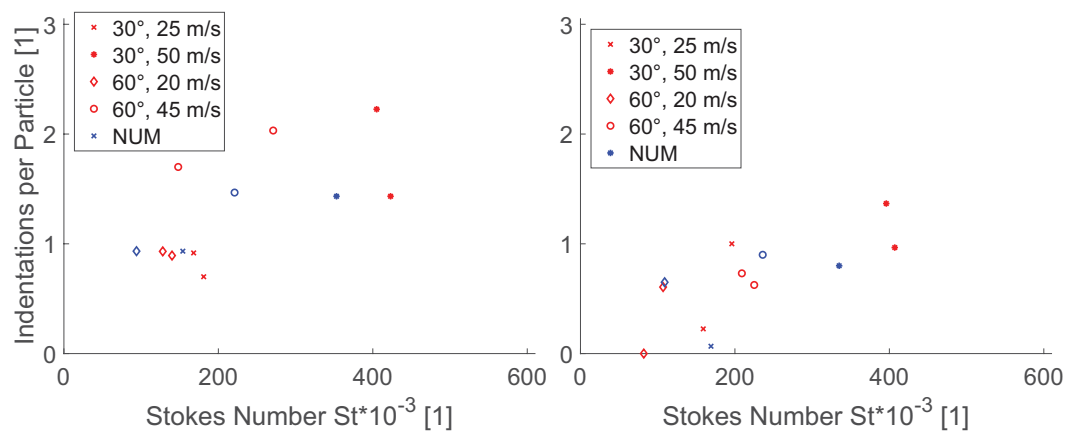

Figure 6: Number of indentations per primary particle from tests with PTFE (left) and SALT (right) - numerical (blue markers) and experimental data (red markers).

particle impacts only partially defoul the PTFE layers. The numerical predictions are comparable to the experimental results and it is therefore concluded that the primary particle indentation behavior as well as secondary particle indentations are generally captured by the model set-up presented.

The next comparison deals with the indentation sizes after the defouling tests and it is presented for PTFE in Fig. 7 and for SALT in Fig. 8. The left-hand display of Fig. 7 shows the mean values and scattering bars for low target angle (i.e. $30^{\circ}$ ) and both nominal air velocities and the right-hand graph shows comparable results from steep target angles (i.e. $60^{\circ}$ ).

In both cases the mean numerical values tend to overpredict the mean experimental outcomes. Good agreement can be seen when comparing the overlapping of the scattering ranges. However, the lower range of indentation diameters is not captured by the simulations. This can be attributed to the actual contribution of very small secondary particles defouling, of which thousands actually exist but just a number is simulated (for details see Rudek [17]). The predictions for steep target angles are closer to the experimental data compared to these for low target angles.
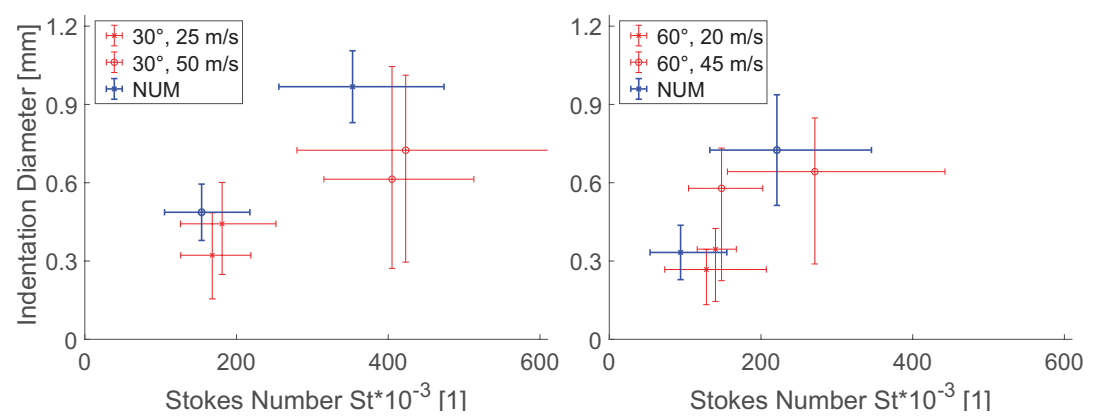

Figure 7: Indentation sizes from tests with PTFE at low (left) and steep target angles (right) - comparison of numerical (blue markers) to experimental data (red markers). 

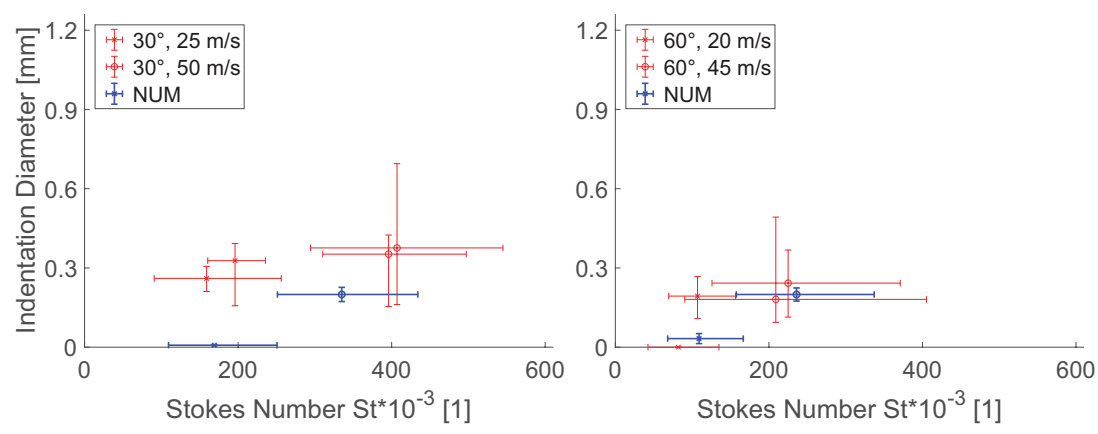

Figure 8: Indentation sizes from tests with SALT at low (left) and steep target angles (right) - comparison of numerical to experimental data.

Results from SALT defouling investigations are shown in Fig. 8 in a comparable representation. The mean numerical results for low target angles clearly underpredict the experimental results. In case of the low nominal velocity there is almost no defouling predicted but there are significant indentations detected in the experiment. The predictions for the higher nominal velocity also underestimate the reality, however these are found to be located in the lower scattering bound of the experimental data. In contrast, the comparison of numerical to experimental data for steeper target angle indentations, Fig. 8, right, shows good agreement of the mean values. The predicted scattering bars of the indentation sizes are much narrower compared to the experimental data. A possible cause for this may be natural scattering experimentally encountered for salt layer defouling, which is not accounted for in the model at the moment (for details see Rudek [17]).

\section{CONCLUSION AND OUTLOOK}

Based on the above results it is concluded that the CFX implementation of the new defouling erosion model, if it is used in conjunction with the new particle breakup model which is not discussed in this communication, adequately predicts actual defouling of PTFE and SALT layers. However, it must be noted that it failed to predict the defouling of SALT in the validation study at low nominal air velocity and low target angle. The lower bounds of the experimental scattering of the PTFE defouling are not captured by the model. In addition the model underpredicts the scatter for SALT defouling. The mean values predicted are in satisfactory agreement with the experimental data for high target angles (mean deviations ca. 15\%) but show significant differences for low target angles (mean deviations ca. 40\%).

The major differences between the PTFE and SALT layer defouling are well predicted for high target angles and those for the low target angle are found to be within the range of experimental scatter, despite the case where the model failed to predict defouling. Furthermore, the particle breakup model used can be seen to be valid in conjunction with the defouling erosion model because secondary particle indentations are predicted when investigating PTFE defouling.

Potential improvement to the defouling erosion model presented can be achieved by the consideration of scattering in the defouling functions. This can be done by introduction of an additional random parameter which must be derived from statistical data processing of the underpinning experiments. Such an additional variable may improve the range of the scatter predicted as well as the prediction of the onset of erosion, which is a constant threshold at the 
moment. This caused the failed SALT layer defouling prediction at low nominal air velocity and low target angle.

In conclusion, both models are considered to be valid for the prediction of axial aircraft compressor defouling simulations and this final application case has been recently addressed in Rudek et al. [16] and in more detail in Rudek [17]. The mean deviations encountered in the validation case study must be kept in mind when discussing results from application case simulations.

Four typical coatings which are relevant to commercial aircraft defouling processes were investigated in the framework of the new model development. It is possible to enlarge this statistical database to numerous coating and particle materials in the future using the basic experiment underpinning the model. Some additional experiments with dry-ice and water-ice particles in conjunction with various additional fouling materials are currently investigated at Hochschule Darmstadt by the authors. It is also planned to enlarge the model to further defouling effects such as thermal and chemical and this is currently addressed in another research project at Hochschule Darmstadt in collaboration with Lufthansa Technik.

\section{REFERENCES}

[1] Ackert, S., Engine maintenance concepts for financiers. Aircraft Monitor, 2, pp. 1-43, 2011.

[2] Kurz, R. \& Brun, K., Fouling mechanisms in axial compressors. Journal of Engineering for Gas Turbines and Power, 134(3), pp. 1-9, 2012. https://doi.org/10.1115/1.4004403

[3] Meher-Homji, C.B. \& Bromley, A., Gas turbine axial compressor fouling and washing.Proceedings of thhe 33rd Turbomachinery Symposium, Vol. 1, pp. 163-192, 2004.

[4] Syverud, E., Brekke, O. \& Bakken, L.E., Axial commpressor deterioration caused by saltwater ingestion. Proceedings of GT2005-ASNME TurboExpo, Reno-Tahoe, USA, Vol. 1, pp. 1-11, 2005.

[5] Meher-Homji, C.B., Compressor and hot section fouling in gas tunbines-causes and effects. Proceedings from the 9th Annual Industrial Energy Technology Conference, Houston, USA, Vol. 1, pp. 261-269, 1987.

[6] Meher-Homji, C.B., Chaker, M.A. \& Motiwala, H.M., Gas turbine performance deterioration. Proceedings of the 30th Turbomachinery Symposium, College Station, USA, Vol. 1, pp. 139-175, 2001.

[7] Martn-Aragn, J. \& Valds, M., A method to determine the economic cost of fouling of gas turbine compressors. Applied Thermal Engineering, 69(1-2), pp. 261-266, 2014.

[8] Diakunchak, I.S., Performance deterioration in industrial gas turbines. International Gas Turbine and Aeroengine Congress and Exposition, Orlando, USA, Vol. 4, pp. 1-8, 1991.

[9] Oosting, J., Boonstra, K., de Haan, A., van der Vecht, D., Stalder, J.P. \& Eicher, U., On line compressor washing on large frame 9-fa gas turbines erosion on $\mathrm{r} 0$ compressor blade leading edge field performance with a novel on line wash system. Proceedings of GT2007: ASME Turbo Expo, Montreal, Canada, Vol. 1, pp. 1-10, 2007.

[10] Mund, F.C. \& Pilidis, P., Gas turbine compressor washing: Historical developments, trends and main design parameters for online systems. Journal of Engineering for Gas Turbines and Power, 128(2), pp. 344-353, 2006. https://doi.org/10.1115/1.2132378

[11] Mund, F.C. \& Pilidis, P., Online compressor washing: a numerical survey of influencing parameters. Proceedings of the Institution of Mechanical Engineers-Journal of Power and Energy, 219(1), pp. 13-23, 2005. https://doi.org/10.1243/095765005x6881 
[12] Brun, K., Grimley, T.A., Foiles, W.C. \& Kurz, R., Experimental evaluation of the effectiveness of online water-washing in gas turbine compressors. Proceedings of the 42nd Turbomachinery Symposium, Houston, USA, Vol. 1, pp. 1-18, 2013.

[13] Syverud, E. \& Bakken, L.E., Online water wash tests of ge j85-13. Proceedings of GT2005-ASME TurboExpo, Reno-Tahoe, USA, Vol. 1, pp. 1-9, 2005.

[14] Giljohann, S., Brutigam, K., Kuhn, S., Annasiri, S. \& Russ, G., Investigations into the on-wing cleaning of commercial jet engines with $\mathrm{co}_{2}$ dry-ice blasting. Deutscher Luftund Raumfahrtkongress, Vol. 1, pp. 1-9, 2012.

[15] Rudek, A., Zitzmann, T., Russ, G. \& Duignan, B., An energy-based approach to assess and predict erosive airfoil defouling. International Journal of Computational Methods and Experimental Measurements: Materials and Contact Characterization, 6(3), pp. 476-486, 2018. https://doi.org/10.2495/cmem-v6-n3-476-486

[16] Rudek, A., Muckenhaupt, D., Kombeitz, R., Zitzmann, T., Russ, G. \& Duignan, B., Experimental and numerical investigation of co2 dry-ice based aircraft compressor cleaning. Proceedings of 13th European Conference on Turbomachinery Fluid Dynamics \& Thermodynamics, Lausanne, Switzerland, Vol. 13, pp. 1-16, 2019.

[17] Rudek, A., Development and Validation of a Numerical Model of the CO2 Dryice Blasting Process for Aircraft Engine Cleaning Applications. Ph.D. thesis, School of Mechanical and Design Engineering, Dublin Institute of Technology, 2018.

[18] Grant, G. \& Tabakoff, W., Erosion prediction in turbomachinery resulting from environmental solid particles. Journal of Aircraft, 12(5), pp. 471-478, 1975. https://doi. org/10.2514/3.59826

[19] Sundararajan, G. \& Shewmon, P.G., The oblique impact of a hard ball against ductile, semi-infinite target materials - experiment and analysis. International Journal of Impact Engineering, 6(1), pp. 3-22, 1987. https://doi.org/10.1016/0734743x(87)90003-0

[20] Hutchings, I.M., Winter, R.E. \& Field, J.E., Solid particle erosion of metals: the removal of surface material by spherical projectiles. Proceedings of the Royal Society of London Series A, Mathematical and Physical Sciences, 348(1654), pp. 379-392, 1976. https:// doi.org/10.1098/rspa.1976.0044

[21] Hutchings, I.M., Macmillan, N.H. \& Rickerby, D.G., Further studies of the oblique impact of a hard sphere against a ductile solid. International Journal of Mechanical Sciences, 23(11), pp. 639-646, 1981. https://doi.org/10.1016/0020-7403(81)90018-7

[22] Tirupataiah, Y. \& Sundararajan, G., A dynamic indentation technique for the characterization of the high strain rate plastic flow behaviour of ductile metals and alloys. Journal of Mechanics and Physics of Solids, 39(2), pp. 243-271, 1991. https://doi. org/10.1016/0022-5096(91)90005-9

[23] Papini, M. \& Spelt, J.K., Indentation-induced buckling of organic coatings part ii: measurements with impacting particles. International Journal of Mechanical Sciences, 40(10), pp. 1061-1068, 1998. https://doi.org/10.1016/s0020-7403(98)00018-6

[24] Kleis, I. \& Hussainova, I., Investigation of particle-wall impact process. Wear, 233-235, pp. 168-173, 1999. https://doi.org/10.1016/s0043-1648(99)00175-1

[25] Papini, M. \& Spelt, J.K., Organic coating removal by particle impact. Wear, 213(1-2), pp. 185-199, 1997. https://doi.org/10.1016/s0043-1648(97)00062-8

[26] Papini, M. \& Spelt, J.K., Indentation-induced buckling of organic coatings part i: theory and analysis. International Journal of Mechanical Sciences, 40(10), pp. 1043-1059, 1998. https://doi.org/10.1016/s0020-7403(97)00147-1 
[27] Gondret, P., Hallouin, E., Lance, M. \& Petit, L., Experiments on the motion of a solid sphere toward a wall: from viscous dissipation to elastohydrodynamic bouncing. Physics of Fluids, 11(9), pp. 2803-2805, 1999. https://doi.org/10.1063/1.870109

[28] Gondret, P., Lance, M. \& Petit, L., Bouncing motion of spherical particles in fluids. Physics of Fluids, 14(2), pp. 643-652, 2002. https://doi.org/10.1063/1.1427920

[29] Barnocky, G. \& Davis, R.H., Elastohydrodynamic collision and rebound of spheres: experimental verification. Physics of Fluids, 31(6), pp. 1324-1329, 1988. https://doi. org/10.1063/1.866725

[30] Davis, R.H., Rager, D.A. \& Good, B.T., Elastohydrodynamic rebound of spheres from coated surfaces. Journal of Fluid Mechanics, 468, pp. 107-119, 2002. https://doi. org/10.1017/s0022112002001489

[31] Wall, S., John, W., Wang, H.C. \& Goren, S.L., Measurements of kinetic energy loss for particles impacting surfaces. Aerosol Science and Technology, 12(4), pp. 926-946, 1990. https://doi.org/10.1080/02786829008959404

[32] Djurovic, B., Jean, E., Papini, M., Tangestanian, P. \& Spelt, J.K., Coating removal from fiber-composites and aluminum using starch media blasting. Wear, 224(1), pp. 22-37, 1999. https://doi.org/10.1016/s0043-1648(98)00308-1

[33] Li, D.Y., Elalem, K., Anderson, M.J. \& Chiovelli, S., A microscale dynamical model for wear simulation. Wear, 225-229, pp. 380-386, 1999. https://doi.org/10.1016/s00431648(98)00368-8

[34] Chen, Q. \& Li, D.Y., Computer simulation of solid particle erosion. Wear, 254(3-4), pp. 203-210, 2003. https://doi.org/10.1016/s0043-1648(03)00006-1

[35] Rudek, A., Russ, G. \& Duignan, B., An experimental and numerical validation study of particle laden supersonic flows. 9th International Conference on Multiphase Flow, Firenze, Italy, 9, pp. 1-6, 2016.

[36] Rudek, A., Russ, G. \& Duignan, B., Particle laden flow investigations in special purpose dry- ice blasting applications. Int International Journal of Computational Methods and Experimental Measurements: Advances in Fluid Mechanics, 4(4), pp. 393-402, 2016. 